\title{
Multiagent Approach to Fuzzy-Linguistic Knowledge Integration
}

\author{
Lukasz Modliński , Grzegorz Popek \\ Wroctaw University of Science and Technology \\ Faculty of Computer Science and Management, Department of Computer Science \\ Wyb. Wyspiańskiego 27, 50-370 Wroctaw, Poland \\ E-mail: \{grzegorz.popek,lukasz.modlinski\}@pwr.edu.pl
}

Received: 15 October 2018; revised: 03 December 2018; accepted: 04 December 2018; published online: 28 December 2018

\begin{abstract}
The paper aims to give at least a partial answer to an urgent need for knowledge processing systems equipped with semantic capabilities. One of the crucial goals is to reflect inner computational models and numerical data outside of the system by presenting linguistic statements easily understood by a non-expert user. The paper follows a motivational scenario and presents a layered approach to knowledge integration. The fundamental rationale behind the proposed approach is that a degree of inconsistency of the whole body of knowledge should be incorporated into the formed summary and conveyed to the external user of the system. The paper deals with a practically important problem of processing modal epistemic statements about an object exhibiting some set of fuzzy properties. The statements represent distributed knowledge of some agent population and are represented on the level of a semi-natural language. In particular, the paper describes an approach to two-level fuzzy-linguistic knowledge integration based on the consensus-theory and clustering methods. In particular, it discusses the difference between the in-cluster level and the cross-cluster level. While this paper considers an environment limited to a single object with multiple properties, it is directly extendable to environments with multiple objects. The reduction is purely technical as it allows for a simplification of a notation and presented descriptions.
\end{abstract}

Key words: knowledge integration, fuzzy-linguistic variable, artificial agents, multi-agent systems, semantic communication

\section{INTRODUCTION}

Data integration topics have been gaining more and more interest lately as the overall amount of knowledge distributed throughout the environment has increased. A similar increase cannot go unnoticed when it comes to the usage of a natural language in communication between the human and the machine or - going even further - between the machines. There is a broad variety of architectures in which the communication process is crucial and any possible improvement of this process may drastically increase the effectiveness of the whole system.

An example of such an architecture is an agent-based system where artificial agents act as independent entities communicating with each other in order to achieve a specific goal. The goal may vary greatly, starting from simple goals such as pure physical effects (e.g. moving objects to a destined area), through an area of coordinational effects (e.g. multi-robot rescue or joint multi-robot mapping in SLAM $[1,2]$ ), towards abstract effects such as multi-agent imitation and knowledge integration (e.g. integration of observational knowledge in multi-robot societies [3]).

Agent-based systems, knowledge integration, and naturallanguage modelling have all been studied separately for years. True problems start when one tries to meaningfully bring the natural-like language into a cognitive artificial system and successfully apply it for a given task. Partially it is caused by numerous conflicts between the researchers regarding various intuitions they base their works on; especially in the field of natural language modelling which is still cursed by the Chomskyan spirit up to this day. In general, the main problem is the lack of a complete cognitive framework implementing semantic capabilities and meaningfully dealing with such problems as Harnad's Symbol Grounding Problem [4]. 
Even though there are many disadvantages and difficulties, the need for knowledge processing systems with semantic capabilities will only ever get stronger. There is a particular need for automation in an area of comprehension and communication using a natural-like language. A variety of real world problems can be described as knowledge integration problems and even relatively uncomplicated solutions ( [5]) form a step forward towards formation of more and more complex systems.

Ideally, artificial knowledge processing systems need to be able to rely on linguistic inputs and outputs. Natural language processing is crucial when it comes to further enrichment of an interaction between the man and the machine. Such systems need to provide capabilities of intuitive humanlike reasoning. Without such capabilities, it is nearly impossible to meaningfully operate based on uncertain or rapidly evolving knowledge often met in real systems.

Despite numerous advantages of such an approach there are also important tradeoffs that have to be taken into account. [6] state that transparency and precision of naturallike languages based systems are dependent on each other and have to be balanced with care. Also the nature of such languages is inherent to inconsistencies which have to be considered while integrating knowledge. These are a direct tradeoff to transparency of linguistic statements which refer to general, subjective interpretations of concepts ( [7]).

In this approach the main goal is to present a distributed knowledge integration process that results with linguistic summarization ( [8]) which is easily understood by a non-expert user. [9] claims that people prefer less precise information stated in a natural language to figures in terms of understandability, therefore the given summary will be stated in a natural-like language. In order to keep inputs form similar to outputs the language of the summary is based on the same natural-like basis.

Natural-like language integration may result with inconsistencies that are unavoidable consdering the nature of such language. In order not to deny the information they hold they have to be taken into account while generating linguistic summary. For that task a representation derived from natural-like languages is employed in this approach.

Finally, an issue worth noting is that nowadays knowledge integrating systems are employed within the units that have limited computing resources. Even though the development of such units has been advanced lately it is still desirable to propose a solution that is simple and lightweight when it comes to computing.

\section{BACKGROUND}

In the recent years the amount of knowledge has increased greatly. Due to vast and extensive growth of internet network the level of knowledge distribution has also greatly increased.
This implies the more noticeable presence of uncertainties in these sources.

Knowledge integration can be seen as a very specific case of knowledge-based reasoning. Specific in the sense that rather than aiming at reaching a particular conclusion based on available knowledge, it aims to give a general description of the available knowledge corpus as a whole.

Existing approaches to knowledge integration base mostly on rule-oriented representations acquired from either machine learning and data mining approaches ( [10]) or domain expert in a standard way. In order to improve operating and reduce the number of required external resources these approaches often employs neural networks or genetic algorithms ( [11]) in reference to rule-based systems. This is to integrate rule sets into a single knowledge base - also in a fuzzy context ( [12]).

Altough rule-based systems have been popular recently popular they struggle to adapt to more complicated, real environments where the knowledge source is greatly distributed and rapid changes may occur as they require either a long retraining process or are negatively affected by exclusive rules in the set which is unavoidable and considered as a fault.

A different approach to knowledge integration may also be applied in a field of recommendation systems ( [13]) in order to provide refined suggestions on the selected domain.

All these approaches share similar properties which one cannot be left unmentioned of. They require a great number of preparations either forming a rule knowledge base providing a reasonable number of rules or equipping considerable and consistent training sets for neural networks initial training. Additional work is to be done also whenever the knowledge source changes over time.

Disadvantages strictly relate to knowledge representation employed in these approaches therefore a different, fuzzybased representation of knowledge has been presented in [14] where rules are exchanged with Fuzzy Cognitive Maps (introduced firstly by [15]) and the integration process is done using Particle Swarm Optimization (PSO) algorithms (introduced in [16], explained widely in [17]) as integration is therefore considered a poorly-structured (ill-structured) problem.

Still these approaches are not flexible enough to grasp more complicated environments as they were proposed for strictly selected domains, especially when it comes to using a natural language as a communication interface between the environment and the system.

\section{PROBLEM DEFINITION}

Although there have been multiple approaches to knowledge integration in recent years, they mostly present rulebased knowledge systems [18]. While such systems do not constitute a problem per se, they tend to require complicated preprocessing [19] before the system can be used in practice. 
What strengthens the issue even more is a fact that such preprocessing has to be redone according to changes in a source of knowledge. In general, implementing rule-based solutions in systems operating in real environments - especially if there is a demand for timely responses - is considered a difficult task.

Another problem with usual knowledge integration systems is an interface between the system and its user. A friendly and easily-interpretable interface for human-machine interaction would obviously form a decent advantage of such a system. The natural candidate for such an interface would be a natural language. In simplistic scenarios, such as evaluating the danger level, color-coded threat levels can be understood faster than full natural language sentences. However, when it comes to an integration of semantic knowledge, natural flexibility and richness of natural language seems irreplaceable to this day.

Our current capabilities of semantic natural language processing are somewhat limited. However, even limited approaches using natural-like language terms or statements can improve the transparency of the system and its behaviour [6].

In particular, the fuzzy-linguistic approach [20-22] - as simplistic as it is in a semantic sense - can be considered a rather successful tackle at modelling of the reality with words. Computational ease and transparency of the approach made it relatively popular and broadly adapted in multiple fields. In some cases, the fuzzy and/or fuzzy-linguistic approach are even considered (overenthusiastically, but still) an approach at the natural language level.

The nature of knowledge integration systems often causes them to work in naturally distributed environments where knowledge comes from external entities [23]. It naturally implies a situation in which the whole body of knowledge is susceptible to inconsistencies. Inconsistent knowledge, if left unhandled, can cause erroneous knowledge integration results and, in general, makes the system prone to failure. Inconsistencies do not only occur due to conflicts between particular agents' knowledge. Inconsistencies are inherent to a natural language, they constitute its inseparable part due to words and sentences holding multiple and/or broad meanings.

Fuzzy-based approaches have been broadly applied in a variety of systems serving as knowledge bases [24] or in order to enhance reasoning and decision-making processes [25]. There are fuzzy-relational databases [26] and fuzzybased database query languages [27] as well. Fuzzy-based approaches and, in particular, the fuzzy-linguistic approach perform well in multiple other tasks and provide an interface for limited natural language inputs.

An interesting scenario occurs when knowledge integration process is implemented in a system gathering data and providing its summaries to non-expert users (e.g. textual summaries of weather [28] or city traffic [29]). Such a situation calls for a certain level of abstraction in a summary as nonexpert users may not understand the meaning behind raw data. A natural candidate for a friendly and intuitively understood solution are natural language-like summaries of data. The already mentioned fuzzy-linguistic approach allows us to form a mapping between observed numerical properties and concepts of a natural language understandable for an external user of the system.

The summarization method for fuzzy relational databases has been proposed by Yager [30], mentioned and extended to interval-based by Niewiadomski in [26]. In his approach Niewiadomski assumes existence of a database with linguistic values upfront which is subject to further summarization in Yager sense. In this approach such assumption has been rejected in favour of gathering data on-fly from other entities taking part in the system using their communication capabilities.

Yager's approach to summarization provides a single summary scoring the highest degree of truth according to a selected quality measurement tool. It may be followed with misleading summaries whenever inconsistencies or incompleteness are present in a database. Inconsistent and incomplete data results in one more problem during knowledge integration. In this approach a cluster analysis is performed before a linguistic summary is formed. In some situations it may be important to notify a user about how "certain" the summary is. It can be done by assigning some numeric value (interpreted as the confidence level - degree of belief) or, to avoid numerical values in the summary, by forming an output as a statement with an auto-epistemic modal operator [31] such as Possible, Believe, Know.

\section{MOTIVATION}

A vast amount of knowledge nowadays requires approaches which are computationally feasible while providing precise, interpretable, and tractable results. The task of an efficient integration is often complicated by the fact that the knowledge may be dynamic (growing or changing in real time), distributed [23], incomplete, and inconsistent.

The approach proposed in this paper emphasized the importance to maintain a general ease-of-use of the system. In order to attain this goal, a solution aimed at mimicking natural language behaviour can be applied during the design of interfaces for handling both output and input of the knowledge processing system. Following this idea, the user does not need to possess an extensive knowledge regarding the particular domain and should be able to communicate with the system using general language concepts. For example, rather than obtaining a row of numerical measurements of various meteorological factors, the user can obtain a textual summary describing the weather in his/her area [28].

Following the above example, the main goal of such a knowledge-integration system is to summarize a distributed body of knowledge in order to provide a fitting, meaningful and easy-to-interpret summary. This allows reducing the need 
of a domain knowledge on an end-user side of the system by providing quick and easy-to-interpret information about a certain environment (e.g. by providing city-traffic descriptions based on numerical data gathered by a distributed network of sensors [5]).

Inconsistencies are one of the characteristics of truly distributed and asynchronous knowledge sources. Unless serious prearrangements are made, inconsistencies are a natural consequence of such a structure of knowledge. A natural way to deal with inconsistencies is to remove them from the data (e.g. by using inputation procedures [32]). While it is usually the most useful way, in some cases it may be important to retain the information about some particular pieces of knowledge being uncertain.

In a natural language humans tend to use auto-epistemic operators [33] to convey their uncertainty. The same can be successfully applied to autonomous systems used to describe data with words. Ultimately, the goal is to inform a recipent of an unclear state of certain parts of knowledge body - completely opposite to a situation in which a recipient remains unaware of potentially faulty information.

Original approaches [34] successfully extend a language generated in artificial agents by applying auto-epistemic operators. However, the setting of distributed knowledge integration is different from the original ones and, in consequence, needs a separate analysis. The closest setting to the one currently investigated can be found in works on generating modally-extended textural descriptions of incomplete city-traffic data [5]. Still, even if it describes a generation of modal descriptions in hierarchical population of agents, it is designed mostly for merging summaries about disjoint regions of the environment and differs from the situation of greatly overlapping and inconsistent knowledge which needs comprehensive reinterpretation.

\section{SYSTEM ARCHITECTURE}

Artificial knowledge integration systems are designed in order to either enhance the knowledge integration process or fully automatize and speed up such a process by removing an expert from the decision making loop. We could draw an analogy to a distinction between decision support systems and decision making systems. Fortunately, typology does not matter here much as long as we accept the crucial idea of artificially supporting humans in tasks which usually need our elaborate participation.

In real-world circumstances integration processes occurs only in environments with knowledge distributed throughout entities forming such environment [23]. Each entity possesses only part of the knowledge. This partial knowledge is usually considered to be subjective, which may lead to possible inconsistencies in the knowledge as a whole.

In this paper we consider an environment limited to a single object with multiple properties. Such reduction allows us to simplify a notation and presented descriptions. Still, the approach can be freely extended to environments with multiple objects. Naturally, it implies that while relations between objects in the world are not out of the question, they are simply omitted by the proposed method. While tackling the problem of inter-object relations would be desirable, to say the least, it is beyond the scope of this step of the research and should be a subject for future consideration.

An ultimate goal of communication is to attain goals of specific agents (attaining goals of the community as a whole can also be modelled as a selfish desire to optimize global 'wellness'). However, the communication process itself can be seen as a way of spreading knowledge in the population, be it acquisition or sharing. Entities in the real-world environment communicate with each other for various reasons. In this process they share their knowledge gathered on environment within which they operate. Such a process is an indispensable part of knowledge integration as it provides information fluctuation and therefore a need for integration.

An artificial system of knowledge integration was mentioned to resemble corresponding real-world processes, therefore an agent-based approach was employed in this work. Intelligent agents are commonly used throughout the literature and the definition of an intelligent agent is presented in [35]. For the sake of simplicity in this approach the architecture was reduced to the minimum of interaction between agents.

In this approach two types of agents may be distinguished, the first being an Observer Agent (OA) focused on conducting observations within real-world environment. OA's goal is to perceive the selected object's properties and pass this information further for integration. The number of such agents depends on various quality and performance factors. A high number of OAs affects the performance as the amount of information increases but it simultaneously raises the quality of the final result.

A recipient of such information is the Main Agent (MA) which is responsible for the integration process. The goal is to integrate the knowledge provided by OA and summarize it in a specific manner in order to release the summary outside of the system.

The division into two types of agents is purely theoretical and is performed in order to outline the goals of each type. In real implementations any agent can possess both an ability to observe the environment and to perform the integration task. However, in this paper it is assumed that these tasks are not performed simultaneously by any particular agent. Although it is also left for future consideration, we claim that possessing its own empirically-verified (but still subjective) knowledge greatly influences the knowledge integration process. Like in humans, private empirical observations of a particular agent take huge precedence over information obtained from others. 


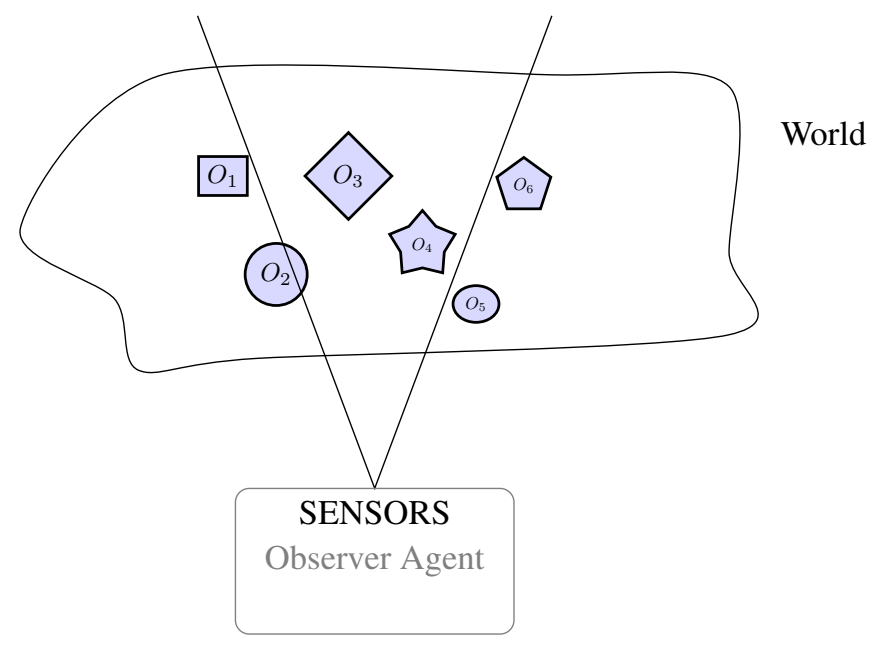

Fig. 1. An agent observing a part of the world

\section{INTEGRATION OUTLINE}

Architecture described in previous paragraphs directly outlines the structure of the investigated integration process. In this particular case the summarization of the distributed knowledge source is an appropriate reason.

In the investigated scenario an input of the integration process consists of distributed sources knowledge in the form of Observer Agents (OAs). Although it may differ in general, it is assumed that OAs are limited to a single observation meaning that an OA collects the values for each unique property of an object once. Were the number of observations more differentiated, the result of the proposed approach would favour agents sending their 'opinions' more often. Sending 'more' would directly translate towards a higher impact on the final result of the integration.

It is also assumed in our scenario that all OAs are equal. That is, the quality of their observation does not vary from one to another. They are equally insightful and equally prone to making errors when replaced with one another. Naturally, in heterogeneous societies of agents one would need to apply additional trust-based mechanisms [36] in order to diminish the impact of incompetent units.

There is exactly one Main Agent (MA) assigned to the knowledge integration task. Following common-sense, it is assumed that the MA does not take part in an observation process. That would cause a heavy interpretational impact as one's empirical observation takes precedence over knowledge attained from others. Under confirmation bias [37] humans tend to take things they see for granted and follow their thinking tending to ignore alternative possibilities, especially if they are not empirically verified. To put this simply, we believe what we see but only possibly trust in what is told by others.

As mentioned earlier, this distinction between OA and the MA still allows them to possess the same architecture.
Both hardware and software components can be the same. It allows for some extended scenarios where a role of the MA is assumed dynamically depending on the situation and information needs of the user. It should be noted that while heterogeneity of agents is generally allowed, it may lead to other problems such as semantic inconsistencies following from possibly different conceptualizations of the external world in differing agents [38].

In order to properly communicate with one another, agents must be equipped with a common language. While this language may be pre-determined by a system designer, there are approaches for an autonomous development of - not too complicated, but still - languages. An approach called Naming Game [39-41] has been developed for agent-based systems in order to both automatize and autonomize this process.

An outline of the approach to the integration process in this work may be described briefly with the following steps and diagram:

1. OAs observe a selected object from the environment,

2. OAs pass observations through messages to MA,

3. Gathered observations are integrated,

4. Result of integration is summarized linguistically,

5. Modal operators are applied in order to emphasize the certainty,

6. Final result is presented outside of the system.

\section{AGENT INTERNAL MODEL}

Throughout the years, a variety of existing approaches to knowledge integration employed multiple various architectures. Among them, an agent-based architecture deserves a particular interest, as it reproduces such relations between real entities as communication interactions or trust mechanisms. 


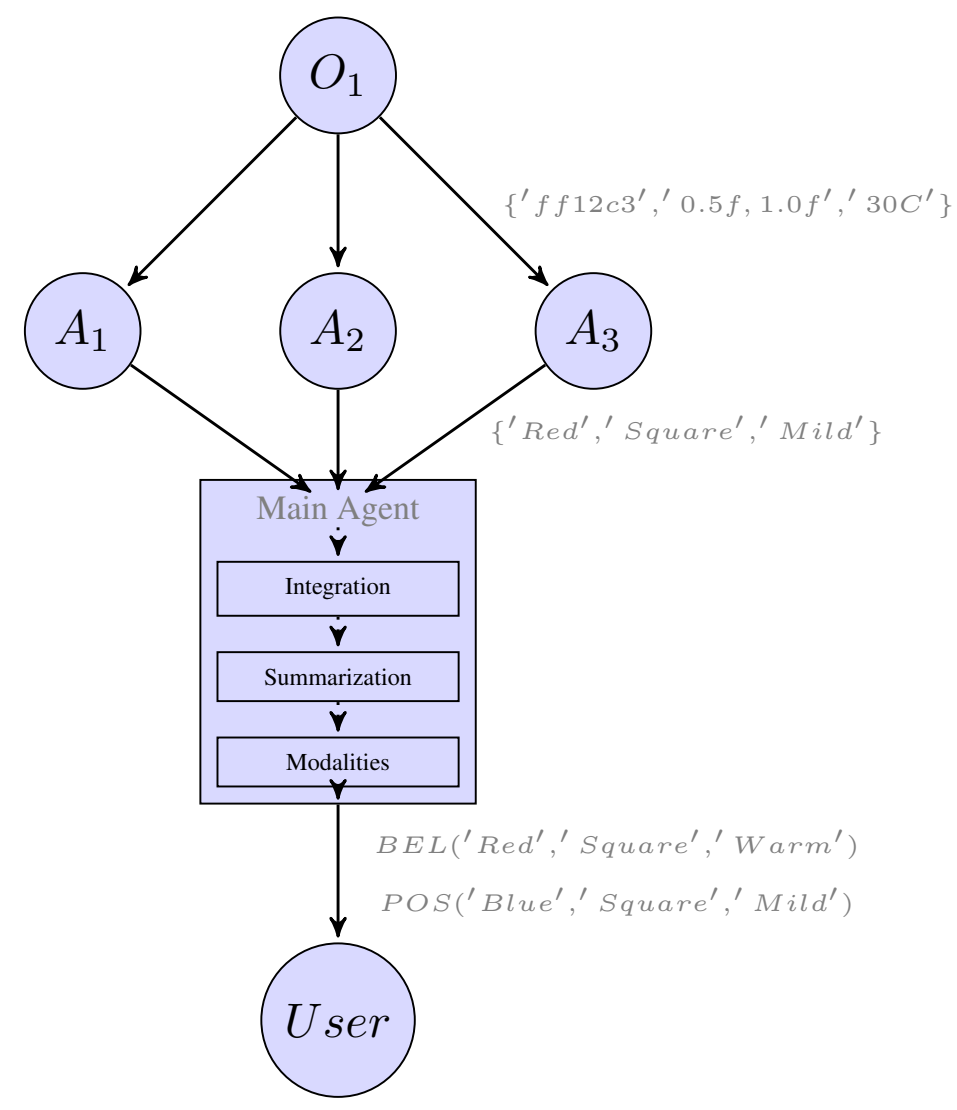

Fig. 2. Simplified steps of the process for a single object, three OAs and three features

A definition of an intelligent and autonomous agent presented in [42] clearly distinguishes the agents from a standard computer software. Another definition that origins back to IBM's Intelligent Agent Strategy white paper states that agent is acting for another, with authority granted by the other.

Autonomous nature of agents is strongly reflected in a pursuit towards achievement of its goals relying on its knowledge partially derived from personal experience. A more detailed discussion on this topic may be found in [43] or [44].

In the proposed approach two different types of agents may be distinguished based on their goals:

1. Observer Agent (OA): An agent whose main goals are to observe and share observations with other agents, especially with the Main Agent. These agents, as a group, constitute a source of distributed knowledge.

2. Main Agent (MA): An agent conducting the integration process as a main goal. This agent also interacts with OAs in order to obtain knowledge for the integration.

\section{1. Observer Agent}

Observer Agent (OA) is one of agents type employed in the presented knowledge integration approach. OA is responsible for conducting an observation of a given object. Existence of multiple OAs observing the environment and producing knowledge naturally implies a distributed nature of the whole system. A distribution is achieved simply thanks to the agent's attributes, ex. autonomous nature, and subjective experience of particular agents. Agents may complement one another by observing different parts of the environment. The system as a whole may also rule out mistakes of single OAs by detecting inconsistencies during the knowledge integration.

As in any intelligent and autonomous agent, there is a set of goals specified for the OA:

1. to observe the given object(s) within the real world and create a corresponding observation vector.

2. to share obtained knowledge with other agents (especially with the Main Agent) using standard communication channels. 


\section{1.1. Internal Architecture}

An internal organisation of any entity in agent-based systems depends mostly on the purpose of such a system. A variety of different architectures were presented in [45] and [46] for distributed medical care and emotion dynamics simulation.

Huang in [45] presents a three-layered knowledge-based architecture of an agent. The domain knowledge layer is responsible for storing knowledge base specific for medical domain, the inference knowledge layer keeps rules related to the domain, and the control knowledge layer applies inferences to domain knowledge whenever new data is available.

In contrast to [45] an architecture in [46] presents an unlayered cognitive agent organisation with components responsible for behaviours, emotions, evaluations, perceptions and knowledge base. Relations between the components are dictated by an algorithm employed in this system to stimulate specific behaviours based on emotions of an agent.

A group of approaches presented in [47] can be identified as layered architectures. Both horizontal and vertical types are described but authors further focus on the latter one referring to InteRRaP agent architecture. In [48] and [49] InteRRaP is stated as a refinement of the head-body-mouth approach to modelling an agent where the layers consecutively are: a reasoning unit, the processing facilities description and a communication unit. On the other hand, a horizontal approach to agent modelling is described in [50]. In that case, three layers are connected simultaneously to the inputs and outputs while working concurrently as they produce activities (and pass them directly to the outputs).

In the proposed approach a vertical layered architecture is employed as a consequence of consecutive parts of the observation process naturally aligning themselves in such order in the system. Each of the layers is responsible for a separate task: either it is retrieving data from sensors, interpreting or forming an observation vector (as presented on Figure 3).

In comparison to architecture in this approach, all mentioned architectures share either a layer or a component related to knowledge (domain-specific knowledge layer in [45], knowledge base component in [46], hierarchical agent knowledge base layer and its components in [47] or in [49], topological map of the various paths and junctions - navigable routes in the world in [50]).

Judging on the description, the position and its connections to other components is a complex element of all the architectures except in [50] where authors are aware of the fact that an agent has to cope with uncertainties and sometimes needs to work having very limited knowledge about the environment.

Mentioned architectures are also equipped with communication components. It conforms with a natural requirement of agents to send out messages of different types either to other systems or, simply, to other agents with which they work.

In contrast to the presented approaches, an architecture described in this paper is much simpler as it is only limited to several behaviours and goals. This allows to fully focus only on required components instead of expanding the architecture for behaviours that may, or may not, be introduced in the future and/or used during operational time of an agent.

All these limitations are made in order to focus on the considered and very specific task-oriented core knowledgeprocessing components and in order to simplify the architecture and decrease the amount of initial knowledge an agent is required to have before he/she is fully prepared to operate in his/her environment.

Every OA is equipped with sensors necessary to retrieve raw data related to object's properties. For the sake of this research an assumption has been made that the vision on the object is not obscured by any mean which would disallow the agent to take observations on the properties he/she is required to. It should also be noted that such an assumption does not necessarily mean that the observations taken by two different agents will be identical. Specific alignment of the sensors and approaches to dealing with observation techniques are not an interest of this research and should be dealt with separately.

In order to fulfil their goals, OAs require a language to communicate with and the communication protocol for sending messages. It is important to note a difference between the language and protocol of communication as the former is crucial in terms of knowledge integration and the latter one is mostly unrelated. Exemplary communication protocols have been presented in [51] and they refer to [52] communication language specification.

In contrast to the communication protocol, a language used by an agent is crucial in terms of successful knowledge sharing. As in any language, entities that communicate among themselves must possess a similar set of concepts. The joint understanding is crucial in successful communication. It is not possible to communicate without shared concepts as it is simply not possible to communicate efficiently using two different languages. Particular internal interpretations related to these concepts are specific for each agent because unlike concepts themselves - they are (or at least, can be [39$41,53])$ derived from their respective experiences and past knowledge.

A requirement for concepts' semantics shared a priori may be considered too strict. In order to partially automatize on-the-run reachability of this requirement, numerous approaches may be used. An exemplary method for achieving a conceptualisation shared throughout all agents is the Naming Game (tNG) described by Steels [40] and Lorkiewicz [39]. In brief, $\mathrm{tNG}$ is a process of drawing attention to an object by naming its characteristic features (or object itself).

The tNG approach has not yet led to creating a fullydeveloped human-like language. Still, it is a good example of how the concepts can be introduced and aligned within agents without interference from their creators (thus fulfilling Zero Semantical Commitment Condition stated for the 
symbol grounding problem in [54]).

It is worth reminding that the multitude of architectures introduced in various papers relate directly to the fact that the proposed architectures are usually goal-oriented. While from time to time there are mentions of new all-capable robots with learning capabilities, in the end - after investigating details - an architecture of an agent is mostly dependent on the processes he/she has to conduct and is employed for.

\section{1.2. Knowledge Gathering Process}

This section describes a process of transforming raw sensorical data into an observation vector. In short, in order to prepare the vector, OA has to use values read from sensors and relate those values to her conceptual knowledge. Based on this internal relation, the OA generates a vector containing symbols of the language describing features of an observed object.

A use of symbols instead of numerical values is dictated by the communication language. This approach reflects a realistic way of semantic communication in which symbols of the language are carriers of subjective (yet shared) meaning instead of objective numerical values.

As shown in Figure 3, this approach translates into an architecture formed of multiple vertically-aligned layers. Each layer corresponds to a distinct stage of an observation process.

An observation process starts with reading values from sensors. It is assumed that every OA is equipped with sensors allowing him/her to read sufficient data on the object. It usually equals to having a separate sensor for each preassumed feature an agent is supposed to observe.

After the values are read, they are processed by the agent in order to obtain an internal subjective interpretation.

Finally, an observation vector is formed for each of the requested features. It is now ready to be shared with others as it is in the form other agents can understand.

In order to avoid tackling an area of particular communication protocols, it is - for simplicity - assumed that the order of features in the vector is fixed and during communication all the agents assume the same predetermined positioning.

\section{2. Main Agent}

Another type of an agent participating in the knowledge integration process is the Main Agent (MA). In contrast to OA, the purpose behind existence of MA is to conduct a process of knowledge integration over a set of observations provided by OAs. Unlike OAs, only one of the agents takes the role of MA within the system.

If integration was performed by multiple agents, it could result in numerous misunderstandings. It follows from a simple fact that their experience may differ from one another (experience in the population may be inconsistent as a whole).

It is also important to note that MA does not participate in the observation process. Otherwise, an approach to integra- tion would greatly differ as it would have to be influenced by the MA view on the object. Having one's own empiricallyverified experience impacts heavily a person's judgement regarding obtained information. We trust more in what we see in comparison to what we are told. In consequence, the personal view of MA dominates over the opinion of others.

As a typical autonomous agent, the MA is equipped with goals to achieve. In this case they are relatively abstract and predefined, and can be stated as follows:

1. Gather observations from Observer Agents.

2. Integrate gathered observations, summarize the result of integration, and deliver it to an external user of this system.

An internal organisation of this agent differs slightly from the architecture of OAs presented in the previous paragraph. Both architectures are described separately but in the end they should be combined within a single agent simply because any agent is supposed to have a capability of taking the role of MA.

Due to the similarity of architectures of MA and OA, similar rules apply. Differences are dictated by a contrast between goals of MA and OA. Other factors affecting the layout of an architecture include:

1. Placement of an agent in a hierarchy of the system.

2. A level of communication language used by MA.

MA relies on the observations gathered from OAs and in consequence - an interface for communication with other agents is required. An assumption has been made here that this interface is fully compatible with OAs communicating protocol and messages delivery rate is $100 \%$. Such an assumption is required in order to focus on the knowledge integration process and to set aside the problems concerning communication itself. More on the topic of communication difficulties and solutions may be found in [51] or [55].

In order to understand the observations of OAs, MA needs to be equipped with a 'similar' language. As it was mentioned in OA paragraph of Section 1, a similar language should be understood as languages being conceptually-consistent. There are multiple ways to ensure MA shares concepts with the OAs. In particular, one can implement tNG mentioned in Section 1.

The function of MA differs from OA which causes an internal organisation to also differ slightly. In MA's architecture consecutive layers of processing correspond to steps of an integration process. In OA, consecutive layers of processing correspond to the observation process, respectively.

Incoming observations are expressed in a language of communication employed in the system. They are reinterpreted into an internal representation using MA's internal semantics. Even though observations made by a particular OA form a base for an original message, the translation process within MA always relates to her own knowledge. This is because of real-life-like assumption that participants of the communication process assume that the meaning is universal amongst themselves. Such re-interpretation has to be 


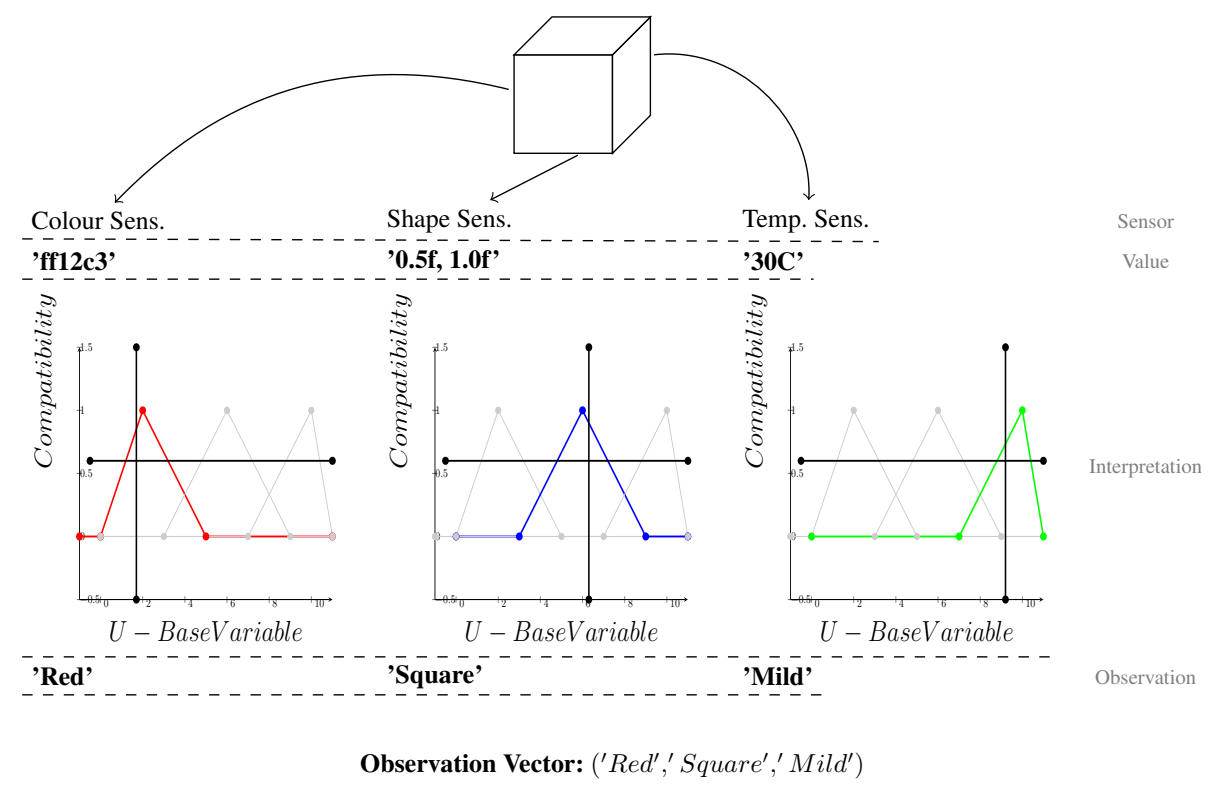

Fig. 3. Observation process

performed in order to initialize the integration process, as it operates on numerical structures instead of symbolic values.

Knowledge integration proceeds as described in Section VI. At this level each of the integration steps corresponds to a specific architecture layer (see. Figure 2).

At this level of integration a process of clustering is conducted in order to provide a full dendrogram ( [56]) required within the next layer. The clustering employs a consensus algorithm [57] and a distance function in order to designate an appropriate representative of a cluster.

After the dendrogram is computed, corresponding clusterisations may be analyzed in order to select the most consistent yet the least numerous clusterisation. Not every integration process results in a single consistent cluster since - as mentioned in VI - observations may be inconsistent. In consequence, the outcome of the integration process should reflect those inconsistencies (that is, it may consist of multiple clusters). The reason behind this is clearly explained in a carpet example presented in [58].

After the cluster analysis, a chosen representative (in a form of a single or multiple clusters) is supposed to be translated back to the linguistic form using the exact same internal representation. This is to aquire a linguistic-level summary of the result in a form conveyable to the external user of the system. Naturally, the translation needs to cover a seemingly unnatural outcome with multiple clusters.

Inconsistencies within the body of knowledge ought to be externally expressed [5]. It is totally unacceptable that the inconsistencies are completely omitted during summarization process.

In order to convey a fact that the original body of knowl- edge is inconsistent, modal statements may be used when forming the final summary. For such cases, the paper [58] proposes an application of auto-epistemic modal operators of possibility, belief and knowledge. The operators (described shortly on VI) reflect the degree of uncertainty of MA regarding the received results of observations (as a whole).

After modality-based statements are assigned, they may be communicated outside of the system.

\section{LINGUSTIC VARIABLES}

Communication with machines has always posed a challenge. Most desirable approaches would employ a natural language for this task as it is widely and commonly used in human-to-human interactions. Various approaches to this matter have been presented lately featuring neural-networks and voice commands processing ( [59]) and group decision making.

Similarly to the mentioned approaches, the proposed solution is natural-like. That is, it employs the Zadehian linguistic variable [20], [21], [22] as a base for a semantic model underlying the communication between agents. Fuzzy-logic-based linguistic variables provide a rational interplay between transparency and precision [6]. Linguistic variables have been successfully applied in a variety of situations, such as the quantitative processes theory [60].

According to [20] the idea behind the linguistic variable (and computing with words, in general) is that words (and complex sentences) are treated as computable values. More formally, the linguistic variable is defined as a following 
quintuple:

$$
(\chi, T(\chi), U, G, M)
$$

where $\chi$ denotes the variable's name; $T(\chi)$ is a set of terms for the variable; $U$ represents a universe of discourse; $G$ is a syntactic rule to generate the terms from $T(\chi) ; M$ is a semantic rule for associating each of the linguistic values from $X \in T(\chi)$ its meaning $M(X)$, where $M(X)$ is a fuzzy subset of $U$. By the meaning of value of the variable we understand its compatibility function $c: U \rightarrow[0 ; 1]$.

The process of communication is conducted using the linguistic terms $T(\chi)$ of the variables as they imitate a naturallike representation of concepts. It should be ensured (as mentioned earlier) that agents require to, at least partially, agree on the meaning of concepts in order to successfully communicate with each other.

Linguistic variables are considered here as a reflection of an agent's internal semantics resulting from the past experiences. These reflections are stored in a form of compatibility function $c: U \rightarrow[0 ; 1]$ for each of concepts from the term set. As an agent acts within the environment they may be modified to adapt accordingly with explorations of the agent. The process of compatibility function forming and evolution is going to be separately investigated in future works.

\section{INTERNAL REPRESENTATION}

Besides the external representation of a communication language, there is an indisputable need for a corresponding (and equivalent!) internal representation of the language. Agents require structures which they are able to naturally 'understand' and process.

The existence of an internal representation within human beings has been discussed yet no consensus has been reached on that matter [61]. Alternative approaches exist (e.g. a representation-less approach has been proposed by Brooks [62]).

Although internal representations allow us to perform more complicated tasks on the knowledge they represent, there are inevitable losses of accuracy when the knowledge is translated to and from this form [6]. These are inevitable tradeoffs justified by the categorization processes as they are an essential part of high-abstraction cognitive beings.

The integration process requires a specific set of operations that are performed on particular internal structures. Let:

$$
\lambda_{X \in T(\chi)} \in[0 ; 1]
$$

In this approach an internal representation is highly related to a compatibility function of $X$, that is:

$$
\bar{X}=\left\{x \in U \Rightarrow c_{X}(x) \geq \lambda_{X}\right\} .
$$

In short, subset $\bar{X}$ contains empirical values for which the compatibility is equal or higher than the activation threshold. $\bar{X}$ is considered as an internal representation of concept $X$.

\section{DISTANCE FUNCTION}

The most important ability required and employed in various phases of the integration process is an ability to compare the observations. Among a variety of methods, a commonsense approach of using a metric function is suitable for this task. The metric needs to be tailored specifically for the form of compared knowledge structures.

Regardless of an assumed form of knowledge, any metric function in any space has to comply with requirements [63]:

1. $\delta(x, x)=0$,

2. $\delta(x, y)=\delta(y, x)$,

3. $\delta(x, y)+\delta(y, z) \geq \delta(x, z)$.

In this particular research, $x$ and $y$ take a form of intervals $x=\left[x_{*} ; x^{*}\right], y=\left[y_{*} ; y^{*}\right]$ where $x_{*}, y_{*}$ and $x^{*}, y^{*}$ denote lower- and upper- bounds of $x$ and $y$, respectively.

Specific metrics suitable for various particular purposes have been presented in the literature. In particular, three following distance functions satisfying mentioned requirements have been proposed [64] for measuring the similarity between finite time-intervals:

$$
\begin{gathered}
\delta_{1}(x, y)=\left\{\begin{array}{l}
\left|x^{*}-y^{*}\right|+\left|x_{*}-y_{*}\right| \\
\max \left\{x^{*}, y^{*}\right\}-\min \left\{x_{*}, y_{*}\right\}
\end{array}\right. \\
\delta_{2}(x, y)=\left\{\left|x^{*}-y^{*}\right|+\left|x_{*}-y_{*}\right|\right. \\
d_{3}(x, y)=\operatorname{card}(x \div y)
\end{gathered}
$$

As mentioned in [65], for a case of a real line $\mathbb{R}$, a distance function can be directly derived from Minkowski's family of distance functions $L_{m}$ designed for Euclidean spaces $\mathbb{R}^{p}$ (naturally, assuming $m=1$ and $p=1$ ). The function $\delta_{2}$ has been analysed and experimented on, while providing promising results in [66] for a case of a knowledge integration process.

Unlike a single-feature scenario, where classical distance functions (like $\delta_{2}$ ) apply, multiple-feature scenarios call for more complicated functions to be designed and employed. Examples on such may be found in [67] where $L_{2}$ was used. An observation vectors have to be normalized in order not to favor any feature over another (due to obvious potential differences in value ranges). Naturally, if particular features are to be favoured, one should stray from normalizing and apply an approach with a vector of weights. 


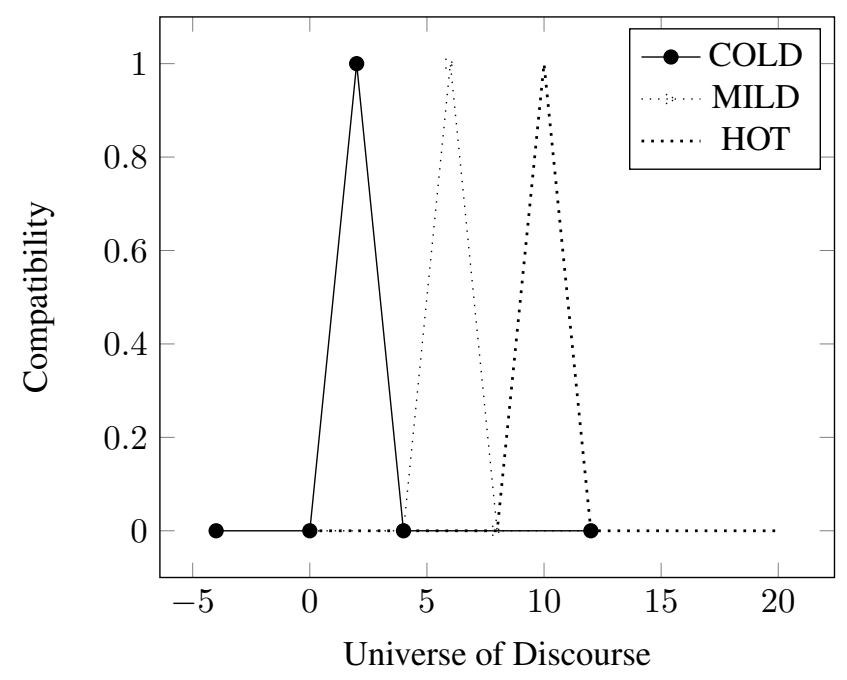

Fig. 4. Example of classic triangular compatibility functions.

\section{CONSENSUS AND CLUSTERING}

\section{1. Consensus}

Integration process may result with a single and easily interpretable outcome. Unfortunately, it often provides a group of similar results which require further processing, e.g. a choice of the final representative [64]. Such a representative is called a consensus. A wider definition and the theory of consensus was presented in [68].

The theory of consensus partially derives from social studies and philosophy where various definitions are presented for politics, epistemology, justice and information amalgamation in individuals $[69,70]$. It was also employed in [71] to allow for a collection of machines to work as a coherent group and to retain an operability under a partial failure.

In an analysed scenario of knowledge provided by a multiagent system, the consensus is applied for the purpose of measuring the distance between whole groups of observations (called clusters). A consensus-based approach is also applied during a translation phase in which the final summary is formed.

A consensus used in an integration process needs to satisfy the following conditions:

1. Consensus resulting from a cluster of elements needs to retain their theoretical-structure (e.g. a consensus evaluated on a set of intervals should be an interval),

2. Consensus may, but does not have to, be equal to any of the elements from an original cluster.

The first condition strays from the general consensus theory [68]. We require the consensus to hold the very same structure as other elements. By this we understand that the number of elements of the vector, their order and their domains are strictly preserved.
Let us consider an example where a set consists of two vectors: ([0;1], ' word $\left.^{\prime}, 15.3\right),([5 ; 10]$, 'sentence', 13.5$)$. Then, a vector $([2 ; 3]$, 'literal', 12.5$)$ could be considered a valid consensus fulfilling the first condition. At the same time, a vector ('literal', $[2 ; 3], 12.5)$ would be considered invalid due to formal requirements.

The second condition is relatively trivial and requires almost no explanation. Simply, whenever the consistency of a set is sufficiently high, the consensus may be found among the elements of the set. In an opposite case (that is, when the consistency is below a desirable threshold), a consensus may differ (in terms of values) from the elements of the set.

Another algorithm for consensus designation was precisely described in [64] where it was paired with the distance function described earlier. It was also used and performed well in [66] in a simple comparison of cluster analysis methods.

After adaptation, an algorithm from [64] takes a form presented in Figure 5.

\section{2. Clustering}

Clustering constitutes the next step specified in an outline of an integration process. It is required for preparation of a set of potential clusterings in order to further analyze their quality (e.g. consistency) and, in consequence, to select the most appropriate one.

A typical goal of a clustering as a data analysis tool is to group similar elements together. There is a multitude of existing solutions tailored specifically for various use cases. A not-so-recent yet still valid, insightful and thorough review of clustering methods can be found in [72].

This paper employs a hierarchical agglomerative clustering [73] in order to accommodate aforementioned needs and 


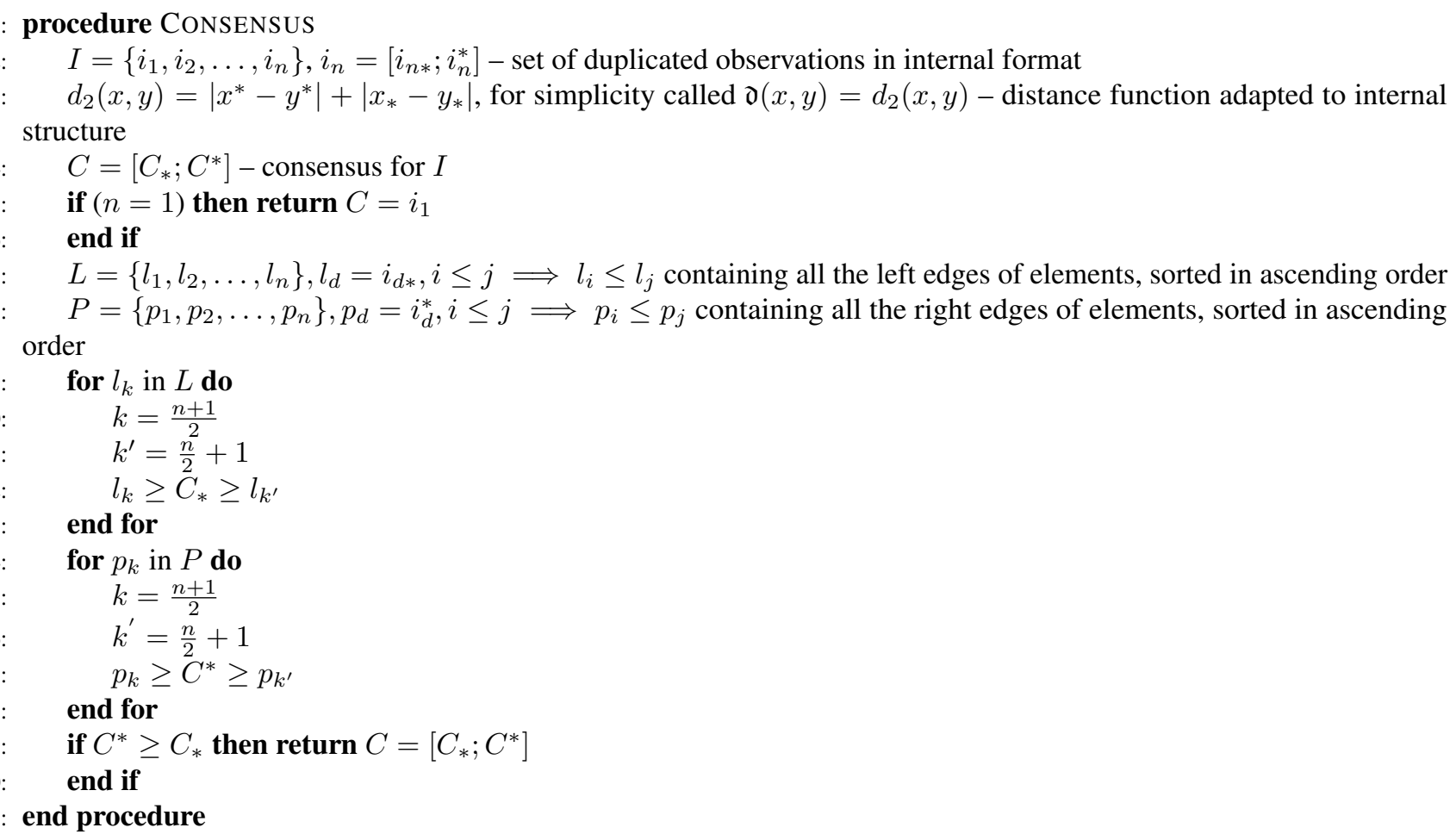

Fig. 5. Algorithm for Consensus Evaluation

generate a full dendrogram of potential clusterings, in contrast to partitional algorithms [72] which provide only a single clustering (with a pre-specified number of clusters, too). Another advantage speaking for an agglomerative approach is its simplicity. It is usually much easier to choose the best candidates for merging rather than find the best split of a cluster. A promising example of applications of hierarchical agglomerative algorithms can be found in the literature [66, 74].

\section{FINAL CLUSTER ANALYSIS}

In order to select a clustering that satisfies the requirements specified within an integration process, a method or algorithm for clustering analysis needs to be provided. Precisely, it is used in order to assess the consistency (and quality, in general) of consecutive clusterings from the dendrogram. The method needs to be suitable for an assumed form of knowledge structures. Multiple approaches are available throughout literature while a well-known method for this task is silhouette [75]. The silhouette algorithm was compared in [66] to a novel cluster analysis method which is Gap Statistics [76] based on comparision to null models [77, 78] that performed better.

This paper employs Gap Statistics. In order to give it a brief introduction, let

$$
D_{r}=\sum_{i, i^{\prime} \in \eta_{n}} d_{i i^{\prime}}
$$

be the summaric distance between consecutive pairs of elements in cluster $K_{r}$ and $d_{i i^{\prime}}$ be the distance function defined earlier in X. Let

$$
W_{k}=\sum_{r=1}^{k} \frac{1}{2 n_{r}} D_{r}
$$

be a within-cluster distribution of elements where $n$ denotes a number of clusters in the clustering and $k$ points at a specific cluster. The idea behind this approach is to maximize a distance between the real data model and its null reference. The Gap distance is given as follows:

$$
\operatorname{Gap}_{n}(k)=E_{n}^{*}\left\{\log \left(W_{k}\right)\right\}-\log \left(W_{k}\right) .
$$

An appropriate references for null models are provided in $[77,78]$ and are not discussed in this work. A more detailed description of gap statistic can be found in [76] where the authors prove a validity of the method.

\section{TRANSLATION}

Integration process provides a numerical summary of gathered knowledge. Achieving a linguistic form of the summary requires additional efforts. In fact, this stage should be seen as a natural inversion of the previous process of translation into an internal representation of an agent. 
Linguistic summaries of data have already been explored under different scenarios in [26] where - in contrast to this approach - data is assumed to be already gathered in a database. It employs a summarization in Yager's [79] sense using degrees of truth for a measurement of the quality [30].

Unlike in mentioned approaches, the quality of summarization in this paper is computed at the numerical level of clustering analysis. The translation stage transforms a numerical output of cluster analysis into concepts used in the communication process.

The translation process can be seen as inversion of an initial interpretation process which converts concepts into a form of an internal representation.

The main intuition behind the proposed approach is depicted in Figure 6. In order to convert numerical summary into a corresponding linguistic form, it needs to be matched against each concept individually. The matching (comparison) follows natural intuitions, e.g.

1. as the numerical result gets more precise, the chance it is covered by the core of a single concept grows,

2. it optimizes the worst-case scenario, i.e., it maximizes the compatibility level at which the whole range of a numerical result is still covered by a respective concept.

The process of matching against a particular concept is intuitively presented in Figure 6 . It starts from the initial value [Figure 6A], pursues towards [Figure 6B] the highest possible compatibility achievable for that particular linguistic concept. Shortly, the numerical representation is pushed as high as possible, but still needs to fully fit within the concept (in a graphical interpretation it fits under the function plot). The process stops once the highest value of compatibility is reached [Figure 6D] for a given numerical representation.

It is also worth noting that the original activation value of concept may be lower than the achieved one [Figure 6C] An intuition underlying this statement is that a precise numerical representation may more adequately fit the given linguistic concept than the one associated with the activation value meant for standarized interpretation purposes. It follows an intuition that albeit the standard activation value governs the concept usage, the essence of the concept itself is usually more compact. It is naturally reflected numerically in fuzzy-linguistic modelling by the still-growing compatibility values. Therefore, a goodness of the match of a summary against a particular concept may be understood as a possibility to bring the said summary as close to the concept core as possible.

Assume the internal representation process is defined as follows:

$$
\Psi_{\rho}\left(\lambda_{\rho}\right)=\left\{x \in U: c_{\rho}(x) \geq \lambda_{\rho}\right\},
$$

where a linguistic variable $\chi$ also refers to a single object's feature and $\rho$ is a linguistic value from the variable's term set $T(\chi)$.
Then translation of the result to its linguistic value depends on a statement:

$$
\bar{\lambda}_{\rho} \in[0 ; 1]: \bar{\Psi} \subseteq \Psi_{\rho}\left(\bar{\lambda}_{\rho}\right) \wedge\left(\forall_{\mathfrak{L}>\bar{\lambda}} \bar{\Psi} \nsubseteq \Psi_{\rho}(\mathfrak{L})\right)
$$

since the process needs to pay attention to such values of activating thresholds which are possibly high while still guaranteeing that the whole $\bar{\Psi}$ is covered by $\Psi_{\rho}\left(\bar{\lambda}_{\rho}\right)$.

In consequence, the value of $\overline{\lambda_{\rho}}$ defines an activation value for $\rho$ on a basis of $\bar{\Psi}$ referred further as Internal Integration Outcome (IIO). It is important to clearly state that the resulting activation value has no connection to the previously defined value used in an internal representation conversion phase.

To fully translate the result of an integration, a translation is conducted on each value of the variable. Let

$$
\left\{\overline{\lambda_{\rho}}: \rho \in T(\chi)\right\}
$$

be the set of activation values for each of the linguistic values from the variable's term set. A selection of the linguistic result for a given variable is then conducted using a formula

$$
\rho^{*}=\arg \max _{\rho \in T(\chi)} \overline{\lambda_{\rho}}
$$

that is, by selecting of a linguistic value paired with the highest activation value.

It is easy to notice that we can reach this result by checking values of membership functions $\mu_{\rho}(u)$ for each particular $\rho$ over the set $\bar{\Psi}$. The minimal value for a particular concept reflects the level up to which we can bring the value of a threshold for this concept. In consequence, by maximizing this value over all concepts and checking which concept allowed us to reach it, we reach the same result:

$$
\rho^{*}=\arg \max _{\rho}\left\{\min _{u \in \bar{\Psi}} \mu_{\rho}(u)\right\} .
$$

Albeit this equation is more direct, it lacks an interpretational transparency of the previously derived method. Still, since it provides us with the same result it should be used in the final implementation.

Given the selection statement it is easy to notice that it may produce more than just a single result. An interpretation of such a situation is relatively straightforward and simply means that multiple concepts describe an IIO with a similar level of adequacy. In consequence, a selection should be based on concepts' ranges. There are two relatively close possibilities:

\section{Similarity-based approach}

$$
\gamma_{\rho}=\frac{\left|\bar{\Psi}_{\rho}\right|}{\left|\Psi_{\rho}\right|}
$$

\section{Distance-based approach}

$$
\gamma_{\rho}=d\left(\Psi_{\rho}, \bar{\Psi}_{\rho}\right) .
$$



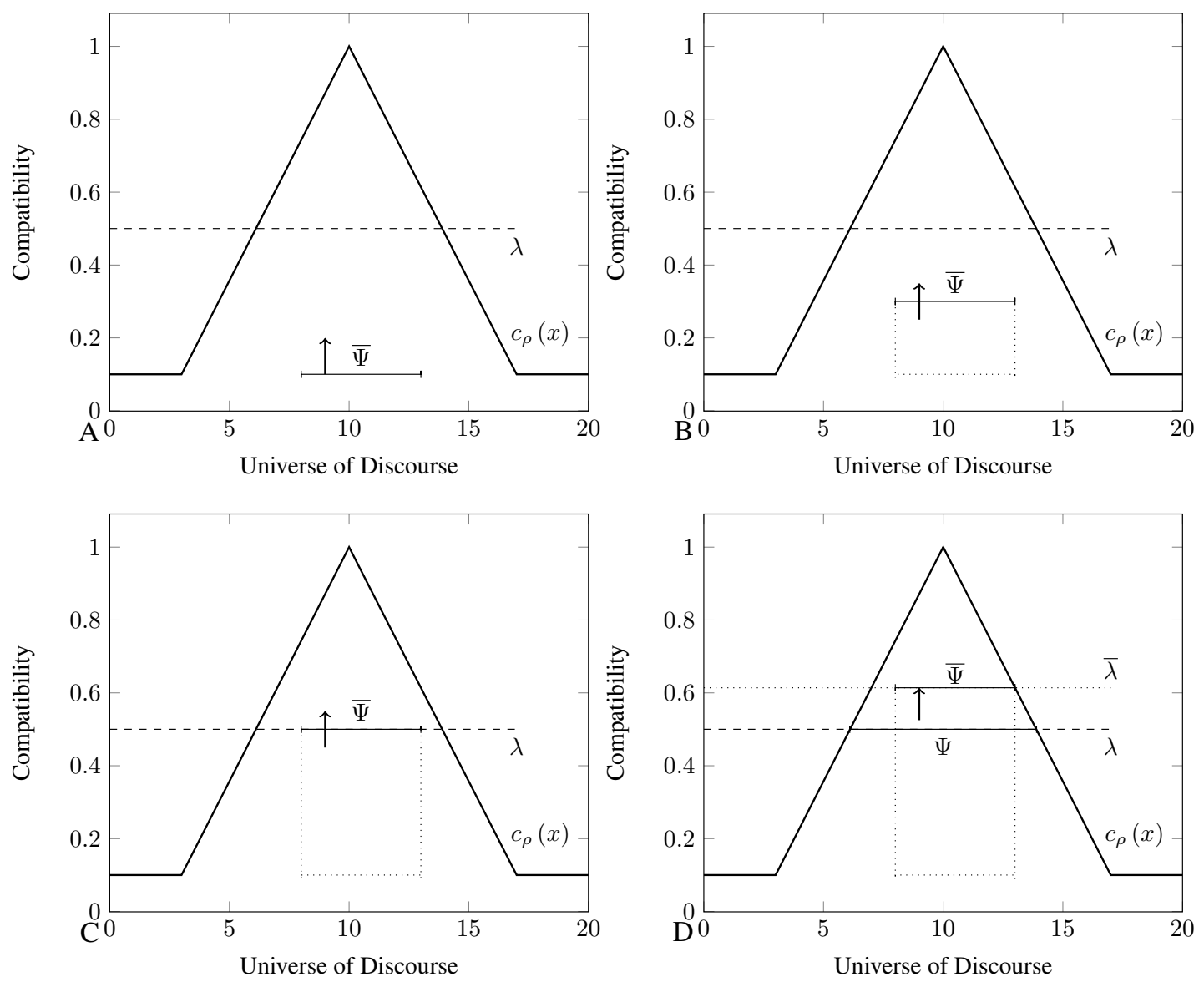

Fig. 6. Translation process graphical interpretation. Consecutive stages: A) initial position; B) moving towards higher compatibility; C) passing the activation value; D) end, maximum available compatibility reached

based on measuring, respectively, either a ratio or a distance between $\bar{\Psi}_{\rho}$ and the whole domain range $\Psi_{\rho}$ achievable at the given level of adequacy $\lambda_{\rho}$. Naturally, depending on the approach, we try to either maximize the similarity or minimize the distance.

\section{MODALITIES}

The translation process results in a linguistic-like summary representing an outcome of the knowledge integration. Despite its linguistic-likeness, the summary does not yet reflect inconsistencies in an originally-distributed knowledge source. In order to enrich an informativeness of the final summary, epistemic modal operators are employed.

An application of epistemic modal operators in linguistic summaries formed by artificial cognitive agents has been advocated in literature ( [53], [34]). A considered enrichment implements the following set of epistemic modal operators:

1. $\operatorname{KNOW}\left(\overline{\rho_{k}}\right)$ with the meaning that linguistic value $\overline{\rho_{k}}$ is known to be the correct interpretation of the integration result.
2. $B E L\left(\overline{\rho_{k}}\right)$ with the meaning that linguistic value $\overline{\rho_{k}}$ is believed to be the correct interpretation of the integration result.

3. $\operatorname{POS}\left(\overline{\rho_{k}}\right)$ with the meaning that linguistic value $\overline{\rho_{k}}$ is possibly the correct interpretation of the integration result.

Natural order of the operators is given by their reflected belief strength, that is

$$
P O S<B E L<K N O W
$$

It is important to note that the modalities may be applied at two different 'levels':

1. 'MACRO' - cross-cluster level - To describe a certainty assigned to the whole cluster, that is, to reflect an interplay between relative strengths of different clusters (from the outcome).

2. 'MICRO' - in-cluster level - To describe a certainty assigned to a single feature of an object (clusterrepresentative).

These two different levels are to be considered separately 
due to differences in various definitions and intuitions related to the meaning and behaviour of modal operators within said levels and due to a further interplay between operators used at different levels.

\section{1. In-Cluster Level}

An in-cluster level could be seen as a clusterrepresentative-interior level. It relates to an internal structure of a representative within a particular cluster.

Two different approaches to grounding modalities are presented for comparative reasons. The first approach presented in [5] can be directly adapted for our purposes. Grounding sets for a given concept $\rho$ can be directly defined as

$$
A_{\rho}=\Psi_{\rho}
$$

and

$$
A_{\neg \rho}=\mathcal{U} \backslash \Psi_{\rho} .
$$

two modified, grounding sets where $\overline{\rho_{z}} \in U$ is an object's $z^{\prime}$ th feature value represented in internal representation, computed in the process of translation and then returned back to internal representation form. These sets hold the activated and non-activated observed values respectively. For comparison purposes a definition of the grounding strength is required.

Originally, the grounding strength is understood as a cardinality of a corresponding grounding set which, in consequence, translates to a following grounding strengths for an activated and non-activated concept:

$$
\begin{gathered}
G_{\rho}=\operatorname{card}\left(A_{\rho}\right), \\
G_{\neg \rho}=\operatorname{card}\left(A_{\neg \rho}\right) .
\end{gathered}
$$

It leads to the following relative grounding strength ${ }^{1}$ :

$$
\mathfrak{L}_{\rho}=\frac{G_{\rho}}{G_{\rho}+G_{\neg \rho}}
$$

for a given concept $\rho$.

A substantial difference proposed in this article is to use $\overline{\lambda_{\rho}}$ as a grounding strength of a given concept $\rho$. It is only natural as, following the assigned interpretation, a value of $\overline{\lambda_{\rho}}$ reflects the compatibility of an Internal Integration Outcome (IIO) with the concept.

This grounding strength is further directly compared against modality thresholds. Different concepts at this level are treated separately and checked for their respective 'semantic fitness'. The relative grounding strength cannot (or should not, to say the least) be computed over all concepts at the same time as their meaning may be interrelated. In particular, there may be some close-to-synonimical concepts with overlapping core regions of meaning. Such concepts may be, according to their shared meaning, activated in a very same situation.

Let us consider an example of concepts with overlapping meaning. It can be relatively easily done in a colour domain. Figure 7 provides hypothetical compatibility functions spanned over an abstract domain for the following colours: ${ }^{2}$

- Magenta,

- FrenchFuchsia,

- Rose.

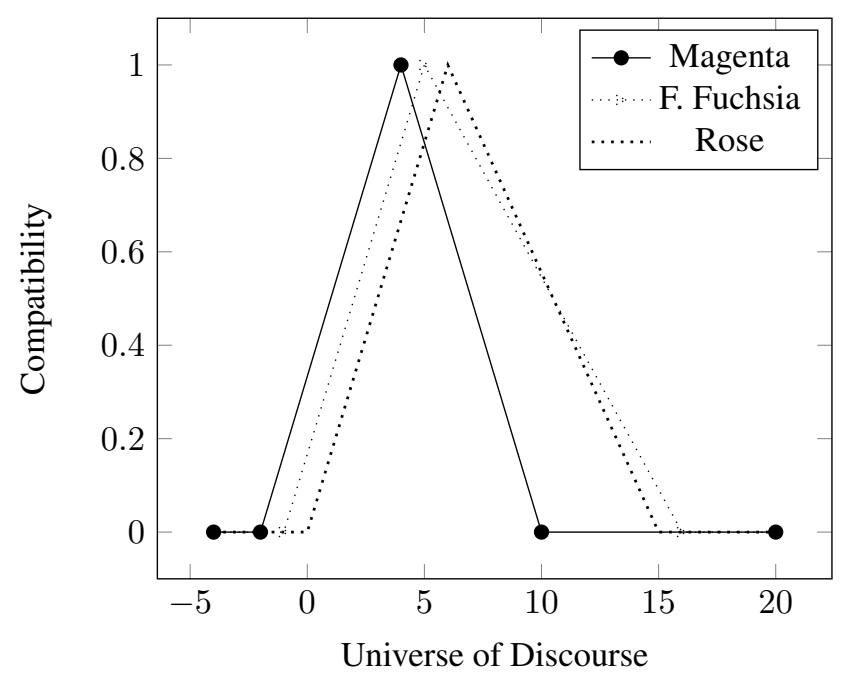

Fig. 7. Compatibilities of three almost overlapping concepts.

And now imagine a bunch of fuchsias. An agent observing the said bouquet may have problems distinguishing between the above three linguistic values. Due to their close meaning and agent's limited colour discerning capabilities, the agent may find the above three both very fitting and at the same time equally viable. In the end, picking one from a set of multiple nearly synonimical terms should not lower the strength of a final modality. The agent may hold a strong belief regarding his/her colour perception while still being unsure about a finally chosen language term.

Even for a set of colours less alike, problems may occur. Consider the following:

- Pink,

- Red,

- Purple.

\footnotetext{
${ }^{1}$ Originally relative grounding strengths and - further - modality levels are denoted using $\lambda$ 's. Due to overlapping notions, these are replaced with $\mathfrak{L}$ 's.

${ }^{2}$ Wiki - Category:Shades of violet.
} 
Very strong correlation between all concepts presented in Figure 8 is undoubtable and clearly perceivable. These colours, while somewhat similar, are unique and separable at higher compatibility levels. Thus, in this scenario all of the above (Pink, Red, Purple) may be highly compatible with the IIO while some being more compatible than others due to their specific, unique properties. In the proposed approach only one concept is going to be selected while others are going to be rejected in favour of that one. Let us assume that the following compatibilities were retrieved during integration and translation processes:

- Pink-0.5

- Red-0.8

- Purple - 0.4

meaning Red is the highest compatibility concept thus will be representing IIO in further subprocesses. While dominating over other concepts Red has a core meaning with both other colors Pink and Purple. This leads to the statement that all these concepts are highly probable based on their shared core meaning while Red has its unique properties that fit the IIO better.

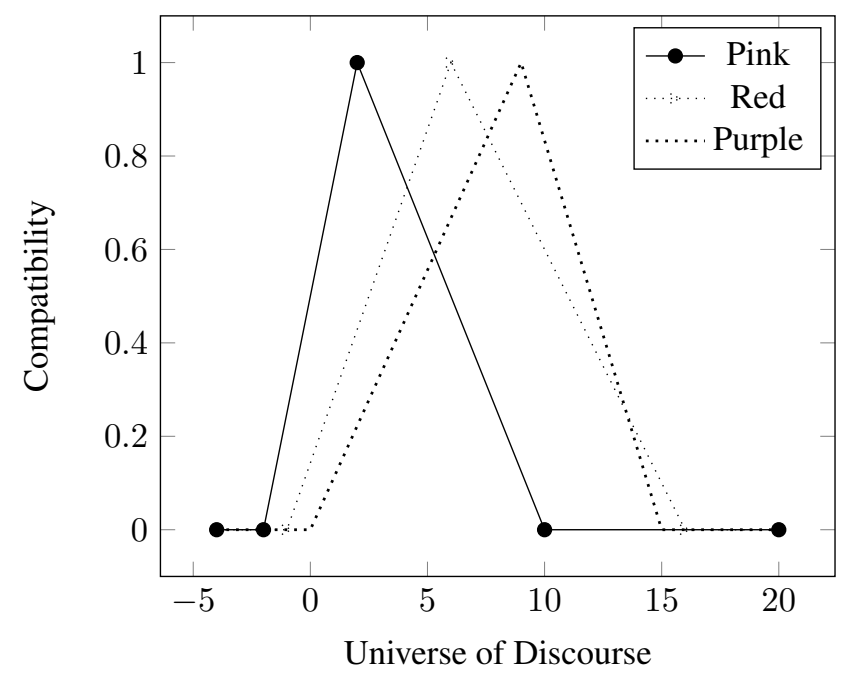

Fig. 8. Compatibilities of less alike colours.

The problem naturally disappears when the concepts are fully-separable in this case and is neglectable for typical concept spaces.

\section{2. Cross-Cluster Level}

In contrast to the In-Cluster level, a Cross-Cluster level uses different definitions of the grounding methods for an evaluation of an adequate epistemic modal operator of possibility $(P O S)$, belief $(B E L)$, and knowledge (KNOW). On this level a cardinality of each cluster is important as it is directly translating into the grounding strength. As it was defined in [58], in order to ground the modal statements on the clustering level the following definition of a grounding strength $\lambda_{n}$ of a cluster $K_{n}$ is required:

$$
\lambda_{n}=\frac{\operatorname{card}\left(K_{n}\right)}{\sum_{j=1} \operatorname{card}\left(K_{j}\right)}
$$

where both $K_{n}$ and $K_{j}$ denote particular single clusters in a clustering $K$ :

$$
K=\left\{K_{1}, K_{2}, \ldots, K_{Z}\right\} .
$$

\section{3. Modality thresholds}

In addition to the order of modality statements, a set of modality thresholds is required in order to assign an adequate modal statement. There is a set of requirements which need to be fulfilled by grounding sets in order to ground a language statement enhanced by a particular auto-epistemic operator. For the case of Zadehian fuzzy-linguistic variable they are described in more detail in [5]. Let us denote a set of modality thresholds as follows

$$
\left(\lambda_{\operatorname{minPos}}, \lambda_{\operatorname{maxPos}}, \lambda_{\operatorname{minBel}}, \lambda_{\operatorname{maxBel}}\right) .
$$

Above four values denote boundaries within which particular modal operators of possibility and belief are used. In order to guarantee that only one modal operator can be chosen at a given time, the intervals $\left[\lambda_{\operatorname{minPos}}, \lambda_{\max P o s}\right)$ and $\left[\lambda_{\min B e l}, \lambda_{\operatorname{maxBel}}\right)$ cannot overlap. Particular requirements used for a choice of the modal operator are defined by a given further set of relations of epistemic satisfaction [31,34].

We assume the following set of requirements for the process of an assignment of a modal operator:

1. It is possible to ground every particular modal operator.

2. The final description of each particular cluster cannot contain more than one modal operator.

3. There can be multiple clusters assigned with an operator POS.

4. Operators $K N O W$ and $B E L$ reflect a dominating view on the observed object. That is, if one of these operators is assigned to particular clusters, none of these two operators can be assigned to any other cluster.

5. The model should allow a situation in which one cluster is assigned with an operator $B E L$ and at least one other cluster is assigned with an operator $P O S$.

6. Modal statements are used only in such cases in which inconsistency [58] is determined. In situations in which the result is consistent an operator of knowledge $(K N O W)$ is assigned.

In order to facilitate these requirements, the following set of inequalities (after [5], [58]) needs to be fulfilled by a set of modality thresholds:

$$
\begin{aligned}
l 0< & \lambda_{\text {minPos }} \leq \min \left\{\frac{1}{\eta}, \frac{1-\lambda_{\operatorname{minBel}}}{\eta-1}\right\} \leq \\
& \leq 0.5<\lambda_{\text {maxPos }}<\lambda_{\text {minBel }}<\lambda_{\text {maxBel }} \leq 1 .
\end{aligned}
$$


In the above formula, $\eta$ denotes the maximal number of operators $P O S$ which can be grounded at the same time, that is, a maximal number of clusters which can be assigned with an operator POS at the same time. An example of a set of modality thresholds fulfilling the above assumptions can be given as follows ${ }^{3}$ :

$$
(0.1,0.6,0.6,0.95)
$$

\section{4. Epistemic satisfaction relations}

In order to attain an assumed overall precision of modalities grounding method, an epistemic satisfaction relation is required for each of the modal operators. The definitions of epistemic satisfaction relations formalize conditions under which a specific modal operator is used. Therefore there are 3 epistemic satisfaction relations - one for each particular operator:

1. Epistemic satisfaction relation $\lambda \rho_{z} \models_{G} \operatorname{KNOW}\left(\overline{C_{l}}\right)$ for in-cluster level and $\lambda_{n} \models_{G} \operatorname{KNOW}\left(\overline{C_{l}}\right)$ for clusterization level occurs whenever $\lambda_{\operatorname{maxBel}}<\lambda_{\rho_{z}} \leq 1$ or $\lambda_{\text {maxBel }}<\lambda_{n} \leq 1$, respectively.

2. Epistemic satisfaction relation $\lambda \rho_{z} \mid={ }_{G} B E L\left(\overline{C_{l}}\right)$ for in-cluster level and $\lambda_{n}=_{G} B E L\left(\overline{C_{l}}\right)$ for clusterization level occurs whenever $\lambda_{\text {minBel }} \leq \lambda_{\rho_{z}}<\lambda_{\text {maxBel }}$ or $\lambda_{\text {minBel }} \leq \lambda_{n}<\lambda_{\text {maxBel }}$, respectively.

3. Epistemic satisfaction relation $\lambda \rho_{z} \models=_{G} \operatorname{POS}\left(\overline{C_{l}}\right)$ for in-cluster level and $\lambda_{n} \models_{G} \operatorname{POS}\left(\overline{C_{l}}\right)$ for clusterization level occurs whenever $\lambda_{\text {minPos }} \leq \lambda_{\rho_{z}}<$ $\lambda_{\operatorname{maxPos}}$ or $\lambda_{\operatorname{minPos}} \leq \lambda_{n}<\lambda_{\operatorname{maxPos}}$, respectively.

\section{MODALITY PROPAGATION}

An extension of linguistic summaries with auto-epistemic modal operators of possibility, belief, and knowledge provides a user of the system with an in-depth insight into the actual uncertainty of information conveyed by the original summary. Originally the operators are independently applied at two distinct levels. In consequence, it is important to consider a relation between these layers when evaluating the final operator to be used within an utterance.

Depending on a focus of attention an agent may intend to describe its knowledge related to either in-cluster level (object's properties) or cross-cluster level (relation between objects). Since both levels co-exist and influence each other, it leads to two types of modality propagations:

1. An ascending propagation - epistemic modal operators on the in-cluster level are propagated onto the crosscluster level.

2. A descending propagation - epistemic modal operators on the cross-cluster level propagated onto the in-cluster level.
In an ascending propagation an agent focuses mainly in terms of summary - on the relations between objects at the cross-cluster level rather than providing description of their details. In fact, the nature of the said object and the way in which it is perceived by the agent may greatly influence the beliefs formulated at a higher level. Therefore, an agent should initially analyse modal operators at the in-cluster level in order to properly adapt them on a cross-cluster level for the focused object.

In contrast to an ascending propagation, in case of a descending propagation agent's principal intentions focus on providing summary about the objects' specific properties. Therefore, modal operators applied at the in-cluster level are the main point of interest of such agent. For a more detailed summary the agent may include operators from the crosscluster level depending on the specific application.

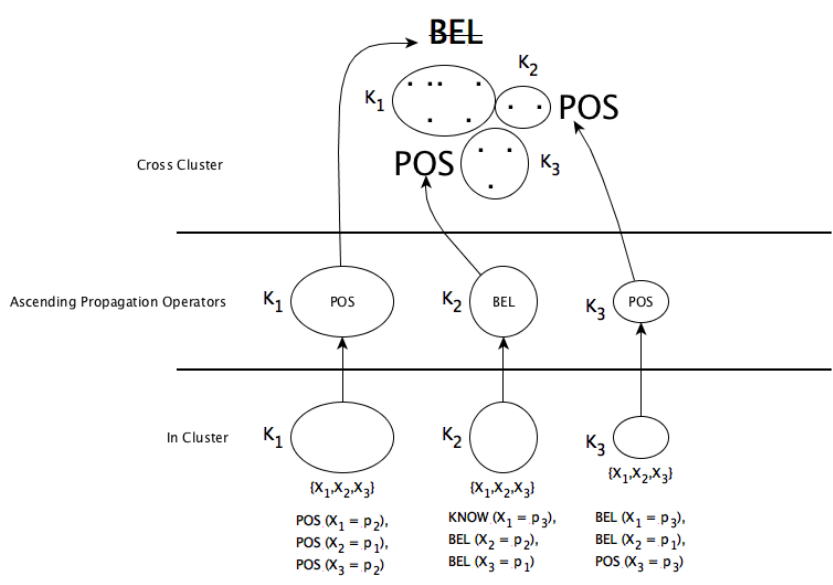

Fig. 9. A choice of a weaker modality in an example of ascending propagation.

Linguistic summaries in the presented research focus on the object as a whole. Therefore, modalities on the crosscluster level form a direct component of a summary and in consequence - ascending propagation is applied. An exemplary approach to ascending propagation is presented in Figure 9. In this situation, the final operator used in an utterance is influenced by the weakest modal operator related to a particular object and present at the in-cluster level.

The corresponding algorithm can be formally described as follows. Each linguistic variable $\chi_{j}$ (that is, each property at the in-cluster level) is assigned with a modal operator $\underline{M}_{j}$ (e.g. $\operatorname{POS}\left(X_{1}=\rho_{2}\right)$ within $K_{1}$ in Figure 9).

Further, given modal operators assigned to all concepts within a given object, the choice of a modal operator to ascend from the in-cluster level follows the equation:

$$
\underline{M}^{*}=\min _{x \in\{1 . . n\}} \underline{M}_{j}
$$

\footnotetext{
${ }^{3}$ This set of values may be unsatisfactory for some strict implementation domains with high values of $\eta$ - for example with $\eta>10$. - but discussions related to $\eta$ are beyond the scope of this paper.
} 
The propagated modality $\underline{M}_{K}^{*}$ assigned to a particular cluster $K$ influences the modality $\bar{M}_{K}$ initially-assigned to this cluster at the cross-cluster level. The final modality $M_{K}^{*}$ is chosen as follows:

$$
\bar{M}_{K}^{*}=\min \left\{\underline{M}_{K}^{*}, \bar{M}_{K}\right\}
$$

This example presents a cautious approach to an ascending propagation where the final modality (used in the summary) is dictated by the weakest of modal operators from both levels. Different applications may use other variations of this approach depending on domain requirements.

\section{FINAL THOUGHTS}

An approach to knowledge integration presented in the paper defines a way to deal with linguistic-based multi-level knowledge integration. Rather than being considered the universal solution, it should be perceived as an insightful study material before designing specific solutions for a particular application. Multiple further extensions are naturally possible, especially taking into consideration the nature of the natural language and its complexity, and they may prove better in particular application areas.

While an approach to fuzzy knowledge integration based on consensus and clustering has been presented, many specific problems remain undiscussed and, in consequence, not fully dealt with. In particular, an example of ascending modality propagation does not relate in any way to the fact that a particular subset of properties may perfectly define a particular object.

The paper considers an environment limited to a single object with multiple properties. Such reduction allowed us to simplify a notation and presented descriptions. Still, the approach can be almost directly extended to environments with multiple objects. Naturally, the direct extension would omit possible relations between real-world objects. While tackling the problem of inter-object relations would be desirable, to say the least, it is beyond the scope of this step of the research and should be left for future consideration.

The process of compatibility function forming and evolution is also going to be separately investigated in future works.

\section{Acknowledgement}

This research was carried out at Wrocław University of Science and Technology (Poland) under Grant 0401/0190/18 titled Models and Methods of Semantic Communication in Cyber-Physical Systems.

\section{References}

[1] C. Luo, A.P. Espinosa, D. Pranantha, A. De Gloria, Multirobot search and rescue team, in Safety, Security, and Rescue Robotics (SSRR), 2011 IEEE International Symposium on. IEEE, 2011, pp. 296-301.

[2] S.B. Williams, O. Pizarro, I. Mahon, M. Johnson-Roberson, "Simultaneous localisation and mapping and dense stereoscopic seafloor reconstruction using an auv," in Experimental robotics, Springer, pp. 407-416 (2009).

[3] A. Jungmann, B. Kleinjohann, W. Richert, Increasing learning speed by imitation in multi-robot societies, in Organic Computing? A Paradigm Shift for Complex Systems, Springer, pp. 295-307, 2011.

[4] S. Harnad, The symbol grounding problem, Physica D: Nonlinear Phenomena 42(1), pp. 335-346 (1990).

[5] G. Popek, Integration of modal and fuzzy methods for agent's knowledge representation, Ph.D. dissertation, Swinburne University of Technology, VIC, Australia, Wroclaw University of Technology, Poland, 2013.

[6] R. Babuška, Construction of fuzzy systems-interplay between precision and transparency, Proc. ESIT 2000, pp. 445-452, 2000.

[7] M. Markovic, Dialectical theory of meaning. Springer Science \& Business Media, 81, 2012.

[8] R.R. Yager, On linguistic summaries of data, Knowledge discovery in databases, pp. 347-363, 1991.

[9] A. Niewiadomski, A type-2 fuzzy approach to linguistic summarization of data, Fuzzy Systems, IEEE Transactions on 16(1), pp. 198-212 (2008).

[10] C.-H. Wang, T.-P. Hong, S.-S. Tseng, A genetics-based approach to knowledge integration and refinement, Journal of Information Science and Engineering 17(1), pp. 85-94 (2001).

[11] C.-H. Wang, T.-P. Hong, M.-B. Chang, S.-S. Tseng, A coverage-based genetic knowledge-integration strategy, Expert Systems with Applications 19(1), pp. 9-17 (2000).

[12] C.-H. Wang, T.-P. Hong, S.-S. Tseng, Integrating membership functions and fuzzy rule sets from multiple knowledge sources, Fuzzy Sets and Systems 112(1), pp. 141-154 (2000).

[13] M. Maleszka, B. Mianowska, N.T. Nguyen, "A method for collaborative recommendation using knowledge integration tools and hierarchical structure of user profiles," Knowledge-Based Systems 47, pp. 1-13 (2013).

[14] K.C. Lee, N. Lee, H. Lee, Multi-agent knowledge integration mechanism using particle swarm optimization, Technological Forecasting and Social Change 79(3), pp. 469-484 (2012).

[15] B. Kosko, Fuzzy cognitive maps, International journal of manmachine studies 24(1), pp. 65-75 (1986).

[16] R.C. Eberhart and J. Kennedy, A new optimizer using particle swarm theory, in Proceedings of the sixth international symposium on micro machine and human science 1. New York, NY, 1995, pp. 39-43.

[17] J. Kennedy, Particle swarm optimization, in Encyclopedia of Machine Learning. Springer, 2010, pp. 760-766.

[18] C.-H. Wang, T.-P. Hong, S.-S. Tseng, Integrating fuzzy knowledge by genetic algorithms, Evolutionary Computation, IEEE Transactions on, 2(4), pp. 138-149 (1998).

[19] B. Jankowska, Using semantic data integration to create reliable rule-based systems with uncertainty, Engineering Applications of Artificial Intelligence 24(8), pp. 1499-1509 (2011).

[20] L.A. Zadeh, The concept of a linguistic variable and its application to approximate reasoning-i, Information sciences 8(3), pp. 199-249 (1975).

[21] L.A. Zadeh, The concept of a linguistic variable and its application to approximate reasoning-ii, Information sciences 8(4), pp. 301-357 (1975). 
[22] L.A. Zadeh, The concept of a linguistic variable and its application to approximate reasoning-iii, Information sciences 9(1), pp. 43-80 (1975).

[23] R. Fagin, J.Y. Halpern, Y. Moses, M.Y. Vardi, Reasoning about knowledge, MIT press Cambridge, 1995, vol. 4.

[24] D. Dubois and H. Prade, On the validation of fuzzy knowledge bases, in Fuzzy Reasoning in Information, Decision and Control Systems, Springer, 1994, pp. 31-49.

[25] H. Bandemer, Fuzzy local inference in fuzzy knowledge bases, in Fuzzy Approach to Reasoning and Decision-Making, Springer, 1992, pp. 39-49.

[26] A. Niewiadomski, Methods for the Linguistic Summarization of Data: Aplications of Fuzzy Sets and Their Extensions, Akademicka Oficyna Wydawnicza Exit, 2008.

[27] N.M. Bigolin and C. Marsala, Fuzzy spatial oql for fuzzy knowledge discovery in databases, in Principles of Data Mining and Knowledge Discovery. Springer, 1998, pp. 246-254.

[28] E. Reiter, S. Sripada, J. Hunter, J. Yu, I. Davy, Choosing words in computer-generated weather forecasts, Artificial Intelligence, 167(1), pp. 137-169 (2005).

[29] G. Popek, R. Kowalczyk, R.P. Katarzyniak, Introducing fuzzy labels to agent-generated textual descriptions of incomplete city-traffic states, in Computational Collective Intelligence. Technologies and Applications. Springer, 2012, pp. 550-561.

[30] R.R. Yager, A new approach to the summarization of data, Information Sciences 28(1), pp. 69-86 (1982).

[31] R.P. Katarzyniak, Grounding crisp and fuzzy ontological concepts in artificial cognitive agents, in Knowledge-Based Intelligent Information and Engineering Systems. Springer, 2006, pp. 1027-1034.

[32] J. Lang and P. Marquis, Removing inconsistencies in assumption-based theories through knowledge-gathering actions, Studia Logica 67(2), pp. 179-214 (2001).

[33] J. Nuyts, Epistemic modality, language, and conceptualization: A cognitive-pragmatic perspective. John Benjamins Publishing, 20015.

[34] R.P. Katarzyniak and A. Pieczyńska-Kuchtiak, "Grounding and extracting modal responses in cognitive agents:'and'query and states of incomplete knowledge," International Journal of Applied Mathematics and Computer Science 14, pp. 249-263 (2004).

[35] L. Padgham and M. Winikoff, Developing intelligent agent systems: A practical guide. John Wiley \& Sons, 200513.

[36] T.B. Klos and J. La Poutré, Decentralized reputation-based trust for assessing agent reliability under aggregate feedback, Software Engineering [SEN], no. E 0422, pp. 1-23, 2004.

[37] M. Lewicka, Confirmation bias, in Personal Control in Action. Springer, 1998, pp. 233-258.

[38] W. Lorkiewicz and R.P. Katarzyniak, Recalling the embodied meaning of modal conjunctions in artificial cognitive agents, in Agent and Multi-Agent Systems: Technologies and Applications. Springer, 2008, pp. 763-772.

[39] W. Lorkiewicz, R. Kowalczyk, R. Katarzyniak, Q.B. Vo, On topic selection strategies in multi-agent naming game, in The 10th International Conference on Autonomous Agents and Multiagent Systems-Volume 2. International Foundation for Autonomous Agents and Multiagent Systems, 2011, pp. 499506.

[40] L. Steels and M. Loetzsch, The grounded naming game, Experiments in cultural language evolution. Amsterdam: John Benjamins, 2012.

[41] P. Vogt, Perceptual grounding in robots, lecture notes on artificial intelligence, in Proceedings of the 6th European Workshop on Learning Robots, Lecture notes on Artificial Intelligence. Springer, 1998.
[42] S. Franklin and A. Graesser, Is it an agent, or just a program?: A taxonomy for autonomous agents, in Intelligent agents III agent theories, architectures, and languages Springer, 1997, pp. 21-35.

[43] G. Weiss, Multiagent systems: a modern approach to distributed artificial intelligence. MIT press, 1999.

[44] S. Russell, P. Norvig, A. Intelligence, A modern approach, Artificial Intelligence. Prentice-Hall, Egnlewood Cliffs 25, 1995.

[45] J. Huang, N.R. Jennings, J. Fox, An agent architecture for distributed medical care, in Intelligent Agents. Springer, 1995, pp. 219-232.

[46] C. Adam, R. Canal, B. Gaudou, H.T. Vinh, P. Taillandier et al., Simulation of the emotion dynamics in a group of agents in an evacuation situation, in Principles and Practice of MultiAgent Systems. Springer, 2012, pp. 604-619.

[47] J.P. Müller, M. Pischel, M. Thiel, Modeling reactive behaviour in vertically layered agent architectures, in Intelligent Agents. Springer, 1995, pp. 261-276.

[48] J.P. Müller and M. Pischel, The agent architecture interrap: Concept and application, 2011.

[49] J.P. Müller, The design of intelligent agents: a layered approach, Springer Science \& Business Media, 1996, 1177.

[50] I.A. Ferguson, Touring machines: An architecture for dynamic, rational, mobile agents, $\mathrm{Ph} . \mathrm{D}$. dissertation, University of Cambridge Cambridge, 1992.

[51] F. Dignum and M. Greaves, Issues in agent communication, Springer Science \& Business Media, 2000, no. 1916.

[52] A. Fipa, Fipa acl message structure specification, Foundation for Intelligent Physical Agents, http://www. fipa. org/specs/fipa00061/SC00061G. html (30.6. 2004), 2002.

[53] R. Katarzyniak, The language grounding problem and its relation to the internal structure of cognitive agents. J. UCS 11(2), pp. 357-374 (2005).

[54] M. Taddeo and L. Floridi, Solving the symbol grounding problem: a critical review of fifteen years of research, Journal of Experimental \& Theoretical Artificial Intelligence 17(4), pp. 419-445 (2005).

[55] F. Dignum, Advances in Agent Communication: International Workshop on Agent Communication Languages ACL 2003, Melbourne, Australia, July 14, 2003, Springer Science \& Business Media, 2004, 2922.

[56] J. Tohá and M. Soto, On the determination of internal nodes of an evolutionary dendrogram, Origins of Life and Evolution of the Biosphere 28(1), pp. 97-103 (1998).

[57] C. Cachin, R. Guerraoui, L. Rodrigues, Introduction to reliable and secure distributed programming, Springer Science \& Business Media, 2011.

[58] W. Lorkiewicz, G. Popek, R.P. Katarzyniak, Intuitive approach to knowledge integration, in Human System Interaction (HSI), 2013 The 6th International Conference on, IEEE, 2013, pp. 40-47.

[59] M. Majewski and W. Kacalak, Natural language humanmachine interface using artificial neural networks, in Advances in Neural Networks-ISNN 2006, Springer, 2006, pp. 1161-1166.

[60] B. D'Ambrosio, Qualitative process theory using linguistic variables, Springer Science \& Business Media, 2012.

[61] T. Matsuka and Y. Sakamoto, A cognitive model of concept learning with a flexible internal representation system, in Advances in Neural Networks-ISNN 2007, Springer, 2007, pp. 1135-1143.

[62] R.A. Brooks, Intelligence without representation, Artificial intelligence 47(1), pp. 139-159 (1991).

[63] K. Kuratowski, A. Mostowski, M. Maczynski, Set theory, North-Holland Amsterdam, 196848. 
[64] N.T. Nguyen, Metody wyboru consensusu i ich zastosowanie w rozwiazywaniu konfliktow w systemach rozproszonych, Oficyna Wydawnicza Politechniki Wroclawskiej, 2002.

[65] H.R.F. Juan Carlos Figueroa-Garci?aa, Yurilev Chalco-Canob, Distance measures for interval type-2 fuzzy numbers, in Discrete Applied Mathematics, Elsvier, 2014.

[66] G. Skorupa, M.L. Katarzyniak, Radoslaw Piotr, M. Mulka, Multi-agent platform for fuzzy structures integration task, ISAT, 2013.

[67] F. d. A. de Carvalho, A fuzzy clustering algorithm for symbolic interval data based on a single adaptive euclidean distance, in Neural Information Processing. Springer, 2006, pp. 10121021.

[68] N.T. Nguyen, Advanced methods for inconsistent knowledge management. Springer, 2007.

[69] K. Lehrer and C. Wagner, Rational Consensus in Science and Society: A Philosophical and Mathematical Study. Springer Science \& Business Media 21. (1981).

[70] K.C. Bausch, The emerging consensus in social systems theory. Springer Science \& Business Media, 2001.

[71] D. Ongaro and J. Ousterhout, In search of an understandable consensus algorithm, in Proc. USENIX Annual Technical Conference, 2014, pp. 305-320.
[72] A.K. Jain, M.N. Murty, P.J. Flynn, Data clustering: a review, ACM computing surveys (CSUR) 31(3), pp. 264-323 (1999).

[73] G. Uchyigit and K. Clark, Hierarchical agglomerative clustering for agent-based dynamic collaborative filtering, in Intelligent Data Engineering and Automated Learning-IDEAL 2004. Springer, 2004, pp. 827-832.

[74] L. Modlinski and G. Popek, Representing result of knowledge integration with modal linguistic statements, ISAT, 2014.

[75] P.J. Rousseeuw, Silhouettes: a graphical aid to the interpretation and validation of cluster analysis, Journal of computational and applied mathematics 20, pp. 53-65 (1987).

[76] R. Tibshirani, G. Walther, T. Hastie, Estimating the number of clusters in a data set via the gap statistic, Journal of the Royal Statistical Society: Series B (Statistical Methodology) 63(2), pp. 411-423, 2001.

[77] A.D. Gordon, Null models in cluster validation, in From data to knowledge. Springer, 1996, pp. 32-44.

[78] N.J. Gotelli and B.J. McGill, Null versus neutral models: what's the difference? Ecography 29(5), pp. 793-800 (2006).

[79] R.R. Yager, K.M. Ford, A.J. Cañas, An approach to the linguistic summarization of data, in Uncertainty in Knowledge Bases. Springer, 1991, pp. 456-468.

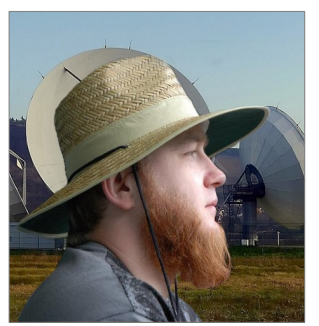

Grzegorz Popek, born 30 September 1983, Wroclaw, Poland; Current Position: Assistant Professor, Wroclaw University of Science and Technology (WrUT), Institute of Informatics, Wroclaw, Poland; Member of Computational Semiotics and Interactive Systems Group, WrUT; Academic Education: 2007: MSc in Computer Science from WrUT, 2013: PhD in Computer Science from WrUT, and Swinburne University of Technology, FICT, Melbourne, Australia; Professional Activities: Organising Committee member of several international conferences (IEA-AIE'08, ICCCI'09, ACIIDS'09, ICCCI'11, ACIIDS'11, ICCCI'12), Member of KES Focus Group on Agent and Multi-agent Systems; Author and co-author of over 20 research journal and conference papers; Current Research Interests: artificial and ambient intelligence - agent and multi-agent systems modelling; artificial cognition - semantic communication, language processing and evolution, computational cognition; knowledge management systems - computational methods in knowledge processing and information retrieval, procedural generation of content in computer games.

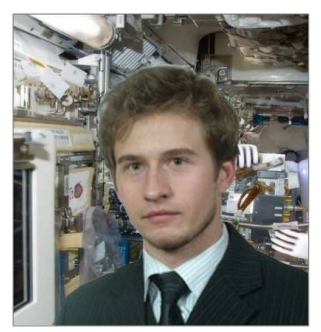

Lukasz Modliński, born 25 July 1990, Wroclaw, Poland; Current Position: PhD Candidate, Wroclaw University of Science and Technology (WrUT), Institute of Informatics, Wroclaw, Poland; Member of Computational Semiotics and Interactive Systems Group, WrUST; Academic Education: 2014: MSc in Computer Science from WrUT; Co-author of 4 conference papers and technical reports; Current Research Interests: artificial and ambient intelligence - agent and multi-agent systems modelling; artificial cognition - semantic communication, knowledge management systems - computational methods in knowledge processing and information retrieval. 